\title{
SURFACE MAGNETOSTRICTION
}

\author{
II. SZYMCZAK AND R. ŻUBEREK \\ Institute of Physics, Polish Academy of Sciences \\ Al. Lotników 32/46, 02-668 Warszawa, Poland
}

\begin{abstract}
Experimental and theoretical research on magnetostriction of nanoscale magnetic multilayers is reviewed. The importance of interfaces and the occurrence of the surface magnetostriction is emphasized. It is shown that the dependence of magnetostriction on the magnetic layer thickness is due to the magnetostrictive strains localized at the interface.
\end{abstract}

PACS numbers: 75.30.Pd, 75.50.Rr, 75.70.Cn

\section{Introduction}

In recent years, the role of surface and interface phenomena in determining the structural, mechanical and magnetic properties of ultrathin layers and multilayers has received an increasing amount of attention. The progress in this field is made mainly due to a tremendous improvement in production and characterization of thin films. Molecular beam epitaxy (MBE) techniques turned out to be particularly useful in production of nanoscale magnetic multilayers (and superlattices) with sharply defined interfaces. In addition to questions involving basic magnetic interactions and phase transitions in 2D systems, these materials are of great technological importance especially in erasable magneto-optic and perpendicular recording applications. Both these applications are based on the concept of surface magnetic anisotropy existing in nanoscale magnetic systems. Strong experimental evidence for the surface anisotropy was found by Gradman [1] (see also [2] and other references therein). Most of the experimental work has been performed on multilayers in which the thickness $t$ of the magnetic layer is varied over a wide range. Since in magnetic materials both magnetic anisotropy and magnetostriction have the same origin, a question arises concerning the magnetostriction of nanoscale magnetic multilayers.

The importance of this problem results from the well-established fact [3] that all metallic multilayers, independent of deposition technique, are in a state of stress. The total stress is composed of thermal stress (due to difference in the thermal expansion coefficient of the two materials) and an intrinsic stress (due to accumulating effect of the crystallographic flow during deposition and also due to electron transfer effects bringing the Fermi energy of two metals closer together [4]). 
Because of the magnetostriction, both kinds of stresses give some contribution to the resulting magnetic anisotropy of the films and multilayers.

In most cases, the magnetostriction of the multilayers is found to change as the thickness $t$ of the magnetic layer is changed [5-10]. The changes in the magnetoelastic properties have been attributed to the magnetostrictive strains which are localized at the interface. Localization of magnetostrictive strains at the surface leads to the linear dependence of the effective magnetostriction constants on the inverse layer thickness $t^{-1}$. Such magnetostrictive effects are called "surface magnetostriction" [6]. A similar situation is observed when one studies the relationship between the interfacial and the elastic response of multilayers. It has been demonstrated [11] that the commonly observed lattice expansion perpendicular to the film plane is not a bulk effect, but is localized at the interface between the contacting metals. It is often claimed $[9,10]$ that the observed changes in elastic and magnetoelastic properties of multilayers are due to the presence of interdiffusion layers which are formed at the interfaces. But in recent years a number of multilayers have been reported to have negligible interface diffusion and considerable surface anisotropy and magnetostriction. Independent experimental evidence for intrinsic character of the surface magnetostriction has been given recently by O'Ilandley and Sun [12-14] who observed giant surface magnetostriction in two amorphous alloys $\left(\mathrm{Fe}_{77} \mathrm{Cr}_{6} \mathrm{~B}_{17}\right.$ and $\mathrm{Co}_{76} \mathrm{Cr}_{4} \mathrm{~B}_{20}$ ) studying the spin polarization of secondary electrons emitted from the surface.

We are going to sum up in this review our experimental data concerning magnetostriction of multilayers. Attention will be focused also on the physical mechanisms responsible for surface magnetostriction.

\section{Experimental technique}

A number of techniques have been developed for measuring the magnetostriction of thin films and multilayers. Among them the most important are:

- the strain modulated ferromagnetic resonance (SMFMR) [5],

- cantilever-capacitance method [8],

- spin polarized secondary electron spectroscopy [14].

It seems that the technique of SMFMR has advantages over the alternative techniques mentioned above. The most conspicuous advantage of SMFMR is the increased sensitivity (magnetostriction constants as small as $10^{-9}$ are detectable).

In the SMFMR technique the strain, periodic in time, applied to the sample placed in a microwave cavity, causes a modulation of the ferromagnetic resonance (FMR) line position. As a consequence of the magnetoelastic coupling, the intensity of the signal obtained after phase-sensitive detection is proportional to the strain modulation depth $m_{\sigma}$. When a magnetic-field modulated FMR signal is simultaneously recorded, the value of $m_{\sigma}$ can be obtained by comparing the intensity of the strain modulated FMR signal $I_{\sigma}$ with that of magnetic-field modulated signal $I_{0}$. For modulation depths much smaller than the linewidth, $m_{\sigma}$ is given by

$$
m_{\sigma}=m_{0} \frac{G_{0}}{G_{\sigma}} \frac{I_{\sigma}}{I_{0}}
$$

where $m_{0}$ is the magnetic field modulation depth and $G_{\sigma}$ and $G_{0}$ are the gains of the amplifier systems for SMFMR and FMR signals, respectively. The shift of 
the resonance line due to the stress $\Delta H_{\sigma}=m_{\sigma}=H_{\sigma}-H_{0}$ contains information on magnetoelastic tensor components. $\Delta H_{\sigma}$ could be calculated by evaluating the resonance fields for the stress $\sigma \neq 0$ and $\sigma=0$ from the ferromagnetic resonance condition [15]:

$$
\left(\frac{\omega}{\gamma}\right)^{2}=\frac{1}{M^{2} \sin ^{2} \Theta}\left[\frac{\partial^{2} F}{\partial \Theta^{2}} \frac{\partial^{2} F}{\partial \phi^{2}}-\left(\frac{\partial^{2} F}{\partial \Theta \partial \phi}\right)^{2}\right],
$$

$\Theta$ being the polar and $\phi-$ the azimuthal angle for magnetization vector $M$. The free magnetic potential energy $F$ consists of Zeeman, demagnetizing, magnetic anisotropy and magnetoelastic parts. The lowest-order phenomenological magnetoelastic contribution to $F$ has the form

$$
F_{\mathrm{me}}=\sum_{i j k l} B_{i j k l} \alpha_{i} \alpha_{j} \varepsilon_{k l},
$$

where $B_{i j k l}$ - magnetoelastic tensor components, $\alpha_{i}$ - the direction cosine of $M$, $\varepsilon_{k l}$ - components of strain tensor. Equation (3) can be rewritten in the alternative form

$$
F_{\mathrm{me}}=\sum_{i j k l} M_{i j k l} \alpha_{i} \alpha_{j} \sigma_{k l},
$$

where $\sigma_{k l}=\sigma \gamma_{k} \gamma_{l}\left(\gamma_{k}\right.$ is the direction cosine of the stress $\sigma$ ) are the components of the uniaxial stress.

It is evident that the symmetry of the multilayers cannot be higher than uniaxial. Assuming, for simplicity, isotropic structure of sublayers, the magnetoelastic tensor $B_{i j k l}$ has the following form:

$$
(B)=\left(\begin{array}{cccccc}
B_{11} & B_{12} & B_{13} & 0 & 0 & 0 \\
B_{12} & B_{11} & B_{13} & 0 & 0 & 0 \\
B_{31} & B_{31} & B_{33} & 0 & 0 & 0 \\
0 & 0 & 0 & B_{44} & 0 & 0 \\
0 & 0 & 0 & 0 & B_{44} & 0 \\
0 & 0 & 0 & 0 & 0 & B_{66}
\end{array}\right),
$$

where $B_{31}=-\left(B_{11}+B_{12}\right), B_{33}=-2 B_{13}, B_{66}=(1 / 2)\left(B_{11}-B_{12}\right)$. The relations between $B_{i j k l}$ and $M_{i j k l}$ are as follows:

$$
\begin{aligned}
& M_{11}=Z\left[\left(c_{11}+c_{12}\right) B_{11}-c_{12}\left(B_{12}+B_{13}\right)\right], \\
& M_{12}=Z\left[\left(c_{11}+c_{12}\right) B_{12}-c_{12}\left(B_{11}+B_{13}\right)\right], \\
& M_{13}=Z\left[\left(c_{11}+c_{12}\right) B_{13}-c_{12}\left(B_{11}+B_{12}\right)\right], \\
& M_{44}=B_{44} / c_{44}, \quad Z^{-1}=\left(c_{11}-c_{12}\right)\left(c_{11}+2 c_{12}\right),
\end{aligned}
$$

where $c_{i j}$ are the elastic constants (in the Voigt notation). In the isotropic approximation

$$
M_{12}=M_{13}=-M_{11} / 2
$$

and the saturation magnetostriction constant

$$
\lambda_{\mathbf{s}}=M_{11}=B_{11} /\left(c_{11}-c_{12}\right) \text {. }
$$




\section{Experimental results}

Magnetostriction has been measured in Co-based and Ni-based multilayers by means of SMFMR [5-7, 16-18] ( see also [19] for details). We consider here in detail experimental data concerning $\mathrm{Ni}$-based magnetic multilayers: $\mathrm{Ni} / \mathrm{Ag}, \mathrm{Ni} / \mathrm{C}$, and $\mathrm{Ni} / \mathrm{Pb}$, since for these systems we succeeded in determination of two independent magnetoelastic constants $M_{11}$ and $M_{12}$ (usually most of the experimental results are given in isotropic approximation; it means that relation $M_{11} / M_{12}=-2$ is assumed).

\section{TABLE}

Magnetostriction constants for $\mathrm{Ni} / \mathrm{Pb}$ multilayers measured at room temperature $\left(\lambda_{\mathrm{s}}\right.$ is calculated in isotropic approximation).

\begin{tabular}{c|c|c|c|c}
\hline \hline$t[\AA]$ & $M_{11} \times 10^{6}$ & $M_{12} \times 10^{6}$ & $M_{11} / M_{12}$ & $\lambda_{\mathrm{s}} \times 10^{6}$ \\
\hline 20 & -5.85 & 2.00 & -2.93 & -5.2 \\
25 & -6.51 & 3.20 & -2.03 & -6.5 \\
30 & -10.05 & 3.41 & -2.95 & -8.9 \\
30 & -10.46 & 3.29 & -3.18 & -9.2 \\
50 & -14.75 & 4.74 & -3.11 & -13.0 \\
70 & -13.80 & 5.36 & -2.58 & -12.7 \\
90 & -12.58 & 5.31 & -2.37 & -12.0 \\
250 & -19.80 & 9.43 & -2.10 & -19.5
\end{tabular}

Table shows the magnetoelastic tensor components for $\mathrm{Ni} / \mathrm{Pb}$ multilayers measured at room temperature. As can be seen from these data, the magnetostriction in measured multilayers is strongly anisotropic $\left(M_{11} / M_{12} \neq-2\right)$. Such behavior is due to the surface magnetostriction which leads to the uniaxial character of the effective magnetostriction in multilayers. The presence of the surface magnetostriction confirms also $\lambda_{\mathrm{s}}\left(t^{-1}\right)$ dependence presented in Fig. 1. This dependence has the following form:

$$
\lambda_{\mathrm{s}}=-17.8 \times 10^{-6}+265[\mathrm{~A}] \times 10^{-6} t^{-1} .
$$

The second term in the dependence (5) is due to surface magnetostriction.

Similar effects were observed in Ni/C (Fig. 2) and Ni/Ag (Fig. 3) multilayers. For $\mathrm{Ni} / \mathrm{C}$ multilayers we have

$$
\lambda_{\mathrm{s}}=-35.9 \times 10^{-6}+910[\mathrm{~A}] \times 10^{-6} t^{-1},
$$

while for $\mathrm{Ni} / \mathrm{Ag}$ the following dependence was measured:

$$
\lambda_{\mathrm{s}}=-35.6 \times 10^{-6}+354[\mathrm{~A}] \times 10^{-6} t^{-1} \text {. }
$$

All of the functions $\lambda_{\mathrm{s}}\left(t^{-1}\right)$ presented in Figs. 1-3 show fairly linear dependence of $\lambda_{\mathbf{s}}$ on the inverse layer thickness of $\mathrm{Ni}$. It indicates the importance of surface (or interface) contributions to effective magnetostriction of multilayers. Some discontinuity observed on the $\lambda_{\mathrm{s}}\left(t^{-1}\right)$ curve for $\mathrm{Ni} / \mathrm{C}$ multilayers (Fig. 2) indicates 


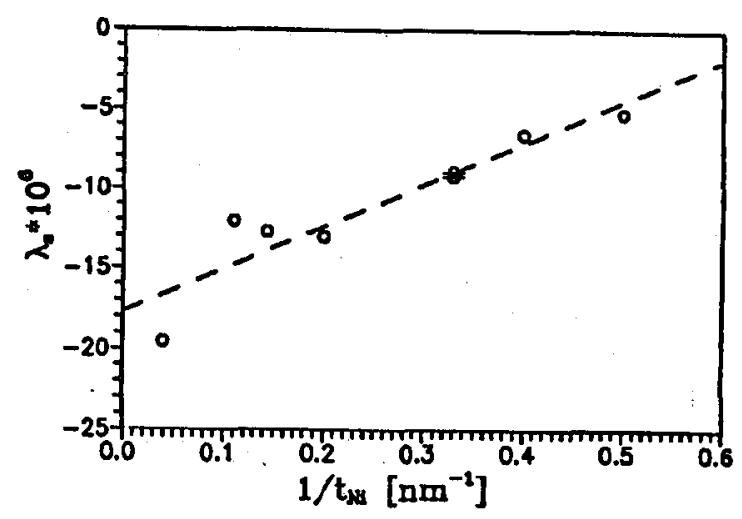

Fig. 1. Magnetostriction constant vs. inverse $\mathrm{Ni}$ layer thickness for $\mathrm{Ni} / \mathrm{Pb}$ multilayer.

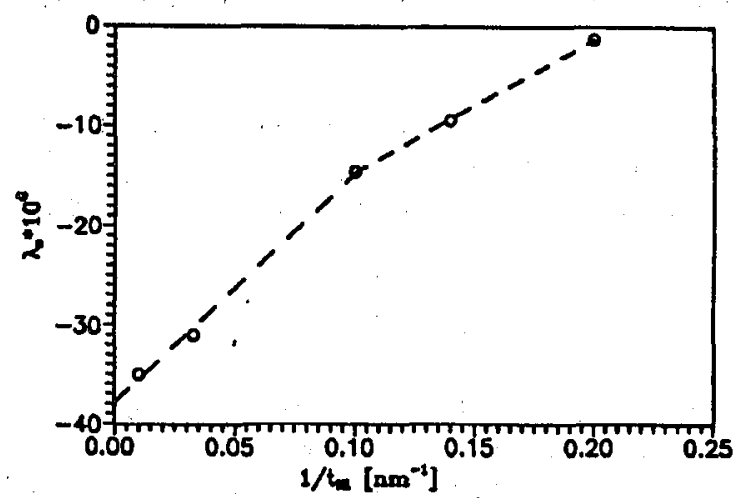

Fig. 2. Magnetostriction constant vs. inverse Ni layer thickness for Ni/C multilayer.

a change in the structure of the film. This was confirmed by electron diffraction which showed indeed that the cubic structure for $\mathrm{Ni}$ was obtained only for $t>60 \AA$ and for thinner layers, $\mathrm{Ni}$ showed a hexagonal structure due to the formation of $\mathrm{Ni}_{3} \mathrm{C}$ phase.

\section{Theoretical models}

At present it is not clear which mechanism is responsible for surface magnetostriction. But since the magnetostriction and magnetic anisotropy have, generally speaking, the same origin, one should expect the following mechanisms to be important in the case of surface magnetostriction:

a) single-ion model based on the spin-orbit interactions,

b) dipole-dipole interactions, 


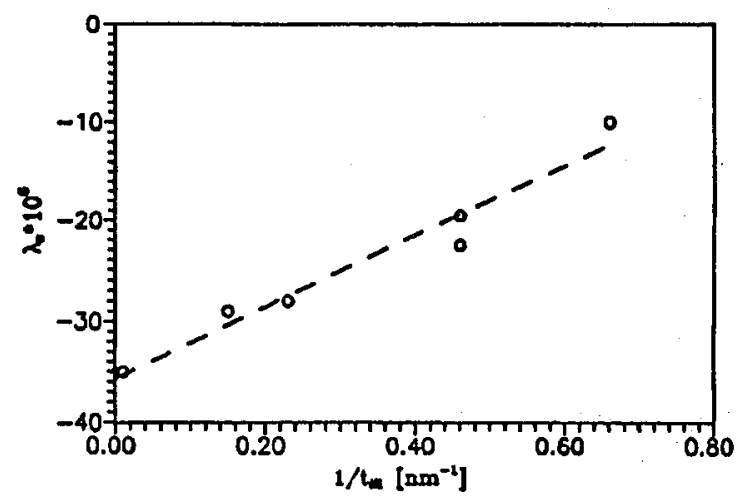

Fig. 3. Magnetostriction constant vs. inverse Ni layer thickness for Ni/Ag multilaycr.

c) nonlinear contributions to bulk magnetoelastic coefficients due to surface strains,

d) surface roughness effects.

At present, detailed theoretical calculations of the surface magnetostriction have been performed only in the frames of dipolar model [20]. In this model the ferromagnetic film is considered as a set of discrete atomic dipoles. The dipolar energy of a dipole $i$ can be expressed as

$$
E^{(i)}=\frac{1}{2} \frac{\mu_{0}}{4 \pi} \sum_{j \neq i}\left[-\frac{m^{2}}{r_{i j}^{3}}+\frac{3\left(m \cdot r_{i j}\right)^{2}}{r_{i j}^{5}}\right],
$$

where $m$ is the magnetic moment of the dipoles, $r_{i j}=r_{i}-r_{j}$ are the relative positions of dipoles $i$ and $j$ and $r_{i j}=\left|r_{i j}\right|$. This dipole-dipole interaction can be interpreted as the energy of the dipole in the field of all other dipoles

$$
E^{(i)}=-0.5 m^{(i)} B^{(i)}, \quad B^{(i)}=\mu_{0} D^{(i)} M_{\mathrm{s}}
$$

where $\boldsymbol{M}_{\mathbf{s}}=m / V_{\text {dip }}$ and $V_{\text {dip }}$ is the volume per dipole. In Cartesian coordinatcs one can write

$$
\begin{aligned}
& \frac{E^{(i)}}{V_{\text {dip }}}=-\frac{1}{2} \mu_{0} M^{2} D_{k l}^{(i)} \alpha_{k} \alpha_{l}, \\
& D_{k l}^{(i)}=\frac{V_{\text {dip }}}{4 \pi} \sum_{j \neq i}\left[-\frac{\delta_{k l}}{r_{i j}^{3}}+\frac{3\left(r_{j i}\right)_{k}\left(r_{i j}\right)_{l}}{r_{i j}^{5}}\right],
\end{aligned}
$$

with $k, l=1,2,3$ and $\alpha_{k}-$ the direction cosines of $M$. The density of magnetoelastic energy is

$$
\frac{E_{\mathrm{me}}^{(i)}}{V_{\mathrm{dip}}}=B_{k l m n}^{(i)} \alpha_{k} \alpha_{l} \varepsilon_{m n}
$$

where

$$
B_{k l m n}^{(i)}=-\frac{1}{2} \mu_{0} M^{2} \frac{\partial D_{k l}^{(i)}}{\partial \varepsilon_{m n}} .
$$

Note that $D^{(i)}$ is symmetric for all positions $i$ and also $D_{11}+D_{22}+D_{33}=0$. 
The actual calculation of $B_{k l m n}^{(i)}$ was performed for various films consisting of monolayers up to several atomic layers. Figures 4 and 5 show the results of $\partial D_{11}^{(i)} / \partial \varepsilon_{11}$ as a function of the layer number $n$ ( $l$ is number of lattice constants) in bcc [001] film and fcc [001] film respectively. It should be emphasized that

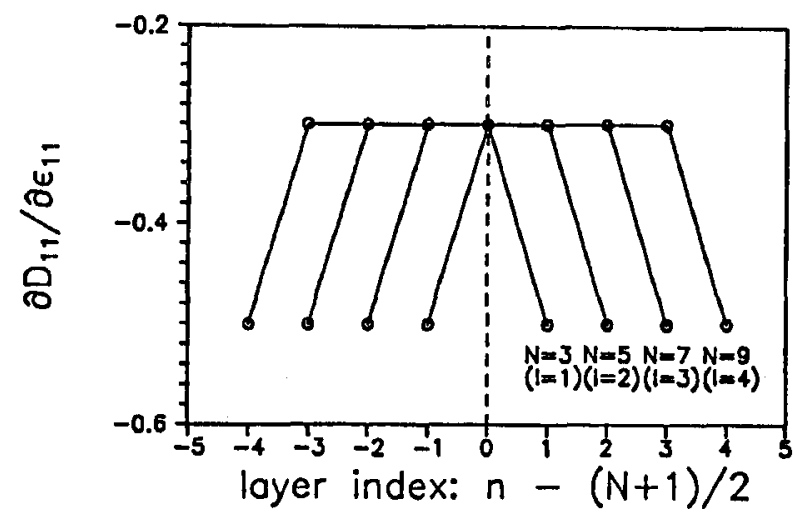

Fig. 4. The dependence of $\partial D_{11}^{(i)} / \partial \varepsilon_{11}$ per atomic layer on the layer number $n$ for bcc [001] film. $N$ is the number of atomic layers in the film.

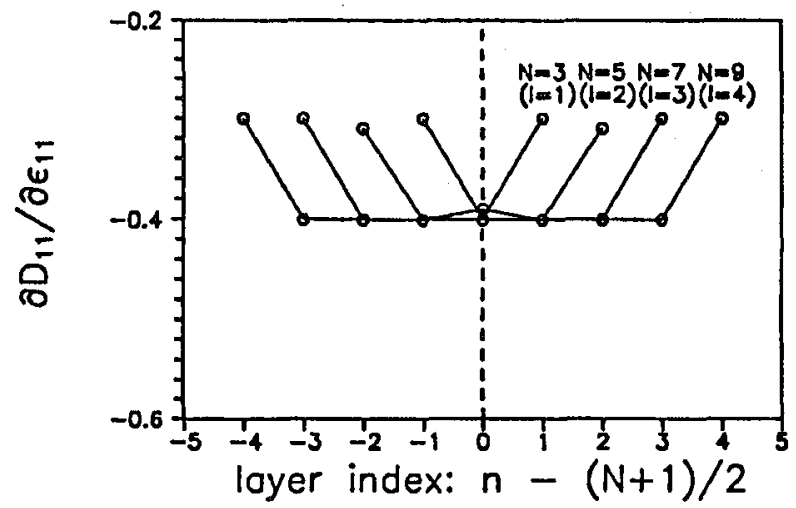

Fig. 5. The dependence of $\partial D_{11}^{(i)} / \partial \varepsilon_{11}$ per atomic layer on the layer number $n$ for fcc [001] film. $N$ is the number of atomic layers in the film.

additional magnetoelastic stresses (represented by $B_{k l m n}^{(i)}$ ) due to limited thickness of the sample are localized at the surface. This result strongly supports the concept of surface magnetostriction. Moreover, it confirms that surface magnetostriction is an intrinsic property of thin films not necessarily arising due to large surface strains.

In Fig. 6 the product of the thickness $t$ (in number of lattice constants) and the average $\partial D_{11} / \partial \varepsilon_{11}$ are plotted as a function of $t$ for the same structures. For 


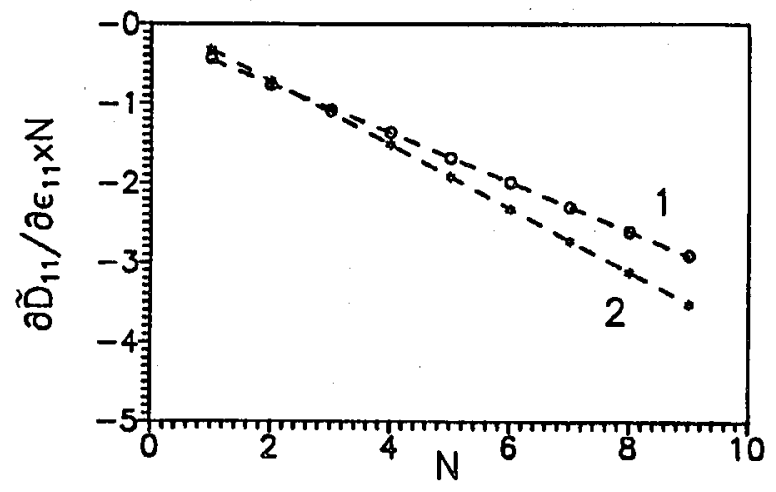

Fig. 6. The dependence of the product of the thickness $t$ (expressed in number of lattice constants) and the average $\partial D_{11} / \partial \varepsilon_{11}$ vs. thickness for bcc [001] film (curve 1) and fcc [001] film (curve 2).

both structures the linear dependence is found in agreement with experimental data [6].

\section{Conclusions}

We have briefly reviewed experimental results and some preliminary theoretical calculations on the surface magnetostriction. It has been emphasized that an increasing number of experimental data is now available. They are roughly consistent with the concept of surface magnetostriction, although some surface modifications during deposition process can act to modify the surface contribution to surface magnetostriction.

\section{Acknowledgment}

This work was supported by the Committee for Scientific Research (grant No. 204959101$)$.

\section{References}

[1] U. Gradman, J. Magn. Magn. Mater. 54-57, 733 (1986).

[2] F.J.A. den Broeder, W. Hoving, P.J.H. Bloemen, J. Magn. Magn. Mater. 93, 562 (1991).

[3] J.A. Huberman, D.W. Hoffmann, Thin Solid Films 171, 5 (1989).

[4] M.L. Huberman, M. Grimsdilch, Phys. Rev. B 46, 7949 (1992).

[5] R. Żuberek, H. Szymczak, R. Krishnan, K.B. Youn, C. Sella, IEEE Trans. Magn. 23, 3699 (1987).

[6] H. Szymczak, R. Żuberek, R. Krishnan, M. Tessier, K.B. Youn, C. Sella, in: ICMFS-12 Conference, Le Creusot (France) 1988, paper TH-03. 
[7] R. Żuberek, H. Szymczak, R. Krishnan, M. Tessier, J. Phys. (France) 49, C8-1761 (1988).

[8] II. Awano, O. Taniguchi, T. Katayama, F. Inoue, A. Itoh, K. Kawanishi, J. Appl. Phys. 64, 6107 (1988).

[9] F.W. Dirne, C.J.M. Denisson, J. Magn. Magn. Mater. 78, 122 (1989).

[10] Y. Nagi, M. Senda, T. Toshima, J. Appl. Phys. 63, 2356 (1988).

[11] B.M. Clemens, G.L. Eesley, Phys. Rev. Lett. 61, 2356 (1988).

[12] R.C. O'Handley, S.W. Sun, Materials Research Society Proceedings, Vol. 231, MRS 1992 , p. 485.

[13] R.C. O'Handley, S.W. Sun, J. Magn. Magn. Mater. 104-107, 1717 (1992).

[14] S.W. Sun, R.C. O'Handley, Phys. Rev. Lelt. 66, 2798 (1991).

[15] J. Smit, H.G. Beljers, Philips Res. Repts. 10, 113 (1955).

[16] R. Żuberek, H. Szymczak, A. Wawro, Mater. Sci. Forum 62-64, 633 (1990).

[17] H. Szymczak, R. Żuberek, R. Krishnan, M. Tessier, IEEE Trans. Magn. 26, 2745 (1990).

[18] R. Żuberek, H. Szymczak, R. Krishnan, H.O. Gupta, C. Sella, M. Kaabouchi, J. Magn. Magn. Mater. 101, 219 (1991).

[19] R. Krishnan, M. Porte, M. Tessier, H. Szymczak, R. Żuberek, Proc. 5lh Int. Conf. on Physics of Magnetic Materials, Eds. W. Gorzkowski, M. Gutowski, H.K. Lachowicz, H. Szymczak, World Scientific, Singapore 1991, p. 294.

[20] T. Szumiata, H. Szymczak, R. Żuberek, INTERMAG'93 - International Magnetics Conference, Stockholm 1993, paper AS-07. 